\title{
Video Case: Sphincterotomy after Small Pre-cut of the Major Duodenal Papilla using Standard Sphincterotome
}

\section{Tarik I Zaher}

Tropical Medicine Department, Faculty of Medicine, Zagazig University, Egypt

\section{Comment}

A 29-year old Egyptian female presented by pain in the right hypochondrium. Abdominal ultrasonography revealed mild dilatation of the common bile duct (CBD). On endoscopic retrograde cholangiopancreatography (ERCP) cannulation of the CBD was difficult.
Small pre-cut for few millimeters upwards starting from the orifice of the major duodenal papilla using the standard sphincterotome was performed .Then Cannulation of the CBD and sphincterotomy were successful. Small stone was extracted from the CBD by balloon . 